University of South Carolina

Scholar Commons

$8-3-2000$

\title{
An Approximation to Miscible Fluid Flows in Porous Media With Point Sources and Sinks by an Eulerian-Lagrangian Localized Adjoint Method and Mixed Finite Element Methods
}

\author{
Hong Wang \\ University of South Carolina - Columbia,wang@math.sc.edu \\ Liang Dong \\ Richard E. Ewing \\ Stephen L. Lyons \\ Guan Qin
}

Follow this and additional works at: https://scholarcommons.sc.edu/math_facpub

Part of the Mathematics Commons

\section{Publication Info}

Published in Siam Journal on Scientific Computing, Volume 22, Issue 2, 2000, pages 561-581. (C) Siam Journal on Scientific Computing 2000, Society for Industrial and Applied Mathematics Wang, H., Liang, D., Ewing, R., Lyons, S., \& Qin, G. (2000). An Approximation to Miscible Fluid Flows in Porous Media with Point Sources and Sinks by an Eulerian--Lagrangian Localized Adjoint Method and Mixed Finite Element Methods. SIAM Journal On Scientific Computing, 22(2), 561-581. doi: 10.1137/ s1064827598349215 inclusion in Faculty Publications by an authorized administrator of Scholar Commons. For more information, please contact digres@mailbox.sc.edu. 


\title{
AN APPROXIMATION TO MISCIBLE FLUID FLOWS IN POROUS MEDIA WITH POINT SOURCES AND SINKS BY AN EULERIAN-LAGRANGIAN LOCALIZED ADJOINT METHOD AND MIXED FINITE ELEMENT METHODS*
}

\author{
HONG WANG ${ }^{\dagger}$, DONG LIANG $^{\ddagger}$, RICHARD E. EWING ${ }^{\S}$, STEPHEN L. LYONS $₫$, AND \\ GUAN QIN 9
}

\begin{abstract}
We develop an Eulerian-Lagrangian localized adjoint method (ELLAM)-mixed finite element method (MFEM) solution technique for accurate numerical simulation of coupled systems of partial differential equations (PDEs), which describe complex fluid flow processes in porous media. An ELLAM, which was shown previously to outperform many widely used methods in the context of linear convection-diffusion PDEs, is presented to solve the transport equation for concentration. Since accurate fluid velocities are crucial in numerical simulations, an MFEM is used to solve the pressure equation for the pressure and Darcy velocity. This minimizes the numerical difficulties occurring in standard methods for approximating velocities caused by differentiation of the pressure and then multiplication by rough coefficients.

The ELLAM-MFEM solution technique significantly reduces temporal errors, symmetrizes the governing transport equation, eliminates nonphysical oscillation and/or excessive numerical dispersion in many simulators, conserves mass, and treats boundary conditions accurately. Numerical experiments show that the ELLAM-MFEM solution technique simulates miscible displacements of incompressible fluid flows in porous media accurately with fairly coarse spatial grids and very large time steps, which are one or two orders of magnitude larger than the time steps used in many methods. Moreover, the ELLAM-MFEM solution technique can treat large mobility ratios, discontinuous permeabilities and porosities, anisotropic dispersion in tensor form, and point sources and sinks.
\end{abstract}

Key words. characteristic methods, Eulerian-Lagrangian methods, miscible fluid flows in porous media with wells, numerical simulation of convection-diffusion equations, reservoir simulation, subsurface contaminant transport

AMS subject classifications. 65M25, 65M60, 76M10, 76S05

PII. S1064827598349215

1. Introduction. Many difficult problems arise in the numerical simulation of complex fluid flow processes in reservoir simulation, subsurface contaminant transport and remediation, and other applications. The mathematical models used to describe these fluid flow processes are coupled systems of nonlinear partial differential equations (PDEs), which are basically convection/diffusion types with convection being the dominant process. Due to the nonlinearity and couplings of these PDEs, the moving steep fronts present in the solutions of these PDEs, the singularities of the solutions at the point sources and sinks (e.g., injection and production wells), and the

* Received by the editors December 14, 1998; accepted for publication (in revised form) March 13, 2000; published electronically August 3, 2000. This research was supported in part by DOE grant DE-FG05-95ER25266, by ONR DEP-EPA-SCoR grant DAAG55-98-1-0002, and by a gift fund from the Upstream Strategic Research Center, Mobil Technology Company, Dallas, TX.

http://www.siam.org/journals/sisc/22-2/34921.html

${ }^{\dagger}$ Department of Mathematics, University of South Carolina, Columbia, SC 29208 (hwang@math.sc.edu).

${ }^{\ddagger}$ School of Mathematics and System Sciences, Shandong University, Jinan, Shandong, 250100, China (dliang@math.sdu.edu.cn).

$\S$ Institute for Scientific Computation, Texas A\&M University, College Station, TX 77843-3404 (ewing@isc.tamu.edu).

`Upstream Strategic Research Center, Mobil Technology Company, 13777 Midway Road, Dallas, TX 75244-4390 (steve_l_lyons@email.mobil.com, guan_gin@email.mobil.com). 
enormous size of field-scale applications, the numerical treatment of these systems often encounters severe difficulties.

Let $c(\mathbf{x}, t)$ be the concentration of an invading fluid, and let $p(\mathbf{x}, t)$ and $\mathbf{u}(\mathbf{x}, t)$ be the pressure and Darcy velocity of the total fluid mixture. The mass conservation equation for the fluid mixture incorporated with the incompressibility condition, Darcy's law, and the mass conservation equation for the invading fluid lead to the following coupled system of PDEs [3, 15], which models the miscible displacement of one incompressible fluid by another in a porous medium reservoir $\Omega$ over a time period of $[0, T]$ :

$$
\begin{array}{rll}
\nabla \cdot \mathbf{u} & =q, & \mathbf{x} \in \Omega, \quad t \in[0, T], \\
\mathbf{u} & =-\frac{\mathbf{K}}{\mu(c)}(\nabla p-\rho g \nabla d), & \mathbf{x} \in \Omega, \quad t \in[0, T], \\
\phi \frac{\partial c}{\partial t}+\nabla \cdot(\mathbf{u} c-\mathbf{D}(\mathbf{x}, \mathbf{u}) \nabla c)=\bar{c} q, & \mathbf{x} \in \Omega, \quad t \in[0, T] .
\end{array}
$$

In many cases, the thickness of the medium is significantly smaller than its length and width. Hence, it is reasonable to average the medium properties vertically and to assume $\Omega \subset \mathbb{R}^{2}$ with a nonuniform local elevation. In $(1.1)-(1.2), \mathbf{x}:=(x, y)$. The dependent variables are the pressure $p(\mathbf{x}, t)$ and the Darcy velocity $\mathbf{u}(\mathbf{x}, t):=$ $\left(u_{x}(\mathbf{x}, t), u_{y}(\mathbf{x}, t)\right)$ of the fluid mixture, where $u_{x}(\mathbf{x}, t)$ and $u_{y}(\mathbf{x}, t)$ are the $x$ and $y$ components of $\mathbf{u}$, respectively, and the volumetric concentration $c(\mathbf{x}, t)$ of the invading fluid. $\mathbf{K}(\mathbf{x})$ is the $2 \times 2$ permeability tensor of the medium, $\mu(c)$ is the concentrationdependent viscosity of the fluid mixture, which is determined by some mixing rule

$$
\mu(c)=\mu(0)\left[(1-c)+M^{\frac{1}{4}} c\right]^{-4},
$$

where $M$ is the mobility ratio between the resident and injected fluids, and $\mu(0)$ is the viscosity of resident fluid (oil). $\rho$ is the density of the fluid mixture, $g$ is the magnitude of gravitational acceleration, $d(\mathbf{x})$ is the reservoir depth, $q(\mathbf{x}, t)$ is the external source/sink term that accounts for the effect of injection and production wells, $\phi(\mathbf{x})$ is the porosity of the medium, $\mathbf{D}$ is the diffusion-dispersion tensor

$$
\mathbf{D}(\mathbf{x}, \mathbf{u}):=\phi(\mathbf{x}) d_{m} \mathbf{I}+\frac{d_{l}}{|\mathbf{u}|}\left(\begin{array}{cc}
u_{x}^{2} & u_{x} u_{y} \\
u_{x} u_{y} & u_{y}^{2}
\end{array}\right)+\frac{d_{t}}{|\mathbf{u}|}\left(\begin{array}{cc}
u_{y}^{2} & -u_{x} u_{y} \\
-u_{x} u_{y} & u_{x}^{2}
\end{array}\right)
$$

where $d_{m}$ is the molecular diffusion coefficient, $\mathbf{I}$ is the $2 \times 2$ identity tensor, and $d_{l}$ and $d_{t}$ are the longitudinal and transverse dispersivities, respectively. $\bar{c}(\mathbf{x}, t)$ is either the specified concentration of the injected fluid at injection wells or $\bar{c}(\mathbf{x}, t)=c(\mathbf{x}, t)$ is the resident concentration at production wells.

In reservoir simulation, the boundary $\Gamma:=\partial \Omega$ is typically impermeable. Consequently, the associated boundary conditions are given by

$$
\begin{aligned}
\mathbf{u} \cdot \mathbf{n} & =0, \quad(\mathbf{x}, t) \in \Gamma \times[0, T], \\
(\mathbf{D} \nabla c) \cdot \mathbf{n} & =0, \quad(\mathbf{x}, t) \in \Gamma \times[0, T] .
\end{aligned}
$$

In addition, the systems (1.1)-(1.2) also need an initial condition for the concentration

$$
c(\mathbf{x}, 0)=c_{0}(\mathbf{x}), \quad \mathbf{x} \in \Omega \text {. }
$$

The combination of the first equation in (1.1) and that in (1.5) leads to the following compatibility condition that must be imposed on the data:

$$
\int_{\Omega} q(\mathbf{x}, t) d \mathbf{x}=0, \quad t \in[0, T] .
$$


Equation (1.7) states that for an incompressible flow with an impermeable boundary, the amount of injected fluid should be equal to the amount that is produced. In addition, (1.1) with the no-flow boundary condition (1.5) can only determine the pressure $p(\mathbf{x}, t)$ up to an additive constant for all the time $t \in[0, T]$. However, this indeterminacy is of no consequence since $\mathbf{u}$ is uniquely determined by Darcy's law, and only $\mathbf{u}($ not $p)$ is needed in (1.2).

2. State of the art in numerical approximations. The principal variable of physical interest in (1.1)-(1.2) is the concentration $c(\mathbf{x}, t)$. In reservoir simulation, it indicates how much of the reservoir is swept by solvent, or equivalently, how much oil is recovered. In subsurface contaminant transport, it shows the movement of the concerned solute in groundwater porous medium flows, which one wants to determine. Because the magnitude of the diffusion-dispersion tensor $\mathbf{D}$ is often much smaller than that of the Darcy velocity $\mathbf{u},(1.2)$ for $c$ is a strongly convection-dominated PDE with small diffusion and dispersion terms indicated by the size of the coefficients $d_{m}, d_{l}$, and $d_{t}$ in (1.4). Moreover, (1.1)-(1.2) are a coupled system of PDEs which is typically defined on a very large physical domain.

2.1. Numerical methods for elliptic pressure PDEs. One important issue in the simulation of porous medium flows is the manner in which the Darcy velocity $\mathbf{u}$ is calculated. Since the convection and diffusion-dispersion terms in (1.2) are governed by the Darcy velocity, accurate approximation to the concentration $c$ requires an accurate approximation to the Darcy velocity $\mathbf{u}$. However, the properties of the porous medium (e.g., K) often change abruptly with sharp changes in lithology. The viscosity $\mu(c)$ also changes rapidly across fluid interfaces. These sharp changes are accompanied by large changes in the pressure gradient which, in a compensatory fashion, yield a fairly smooth Darcy velocity $\mathbf{u}$. Standard finite difference or finite element methods (FDMs, FEMs, respectively) solve (1.1) for the pressure $p$, which is not necessarily smooth due to the impact of the rough coefficients. The resulting $p$ is then differentiated numerically and then multiplied by a possibly rough coefficient to determine the Darcy velocity $\mathbf{u}$. Therefore, these methods generate a rough and often inaccurate velocity $\mathbf{u}$, which then reduces the accuracy of the approximation to (1.2). Mixed finite element methods (MFEMs) $[6,24]$ approximate both $p$ and $\mathbf{u}$ from the system (1.1) simultaneously, yield an accurate velocity field $\mathbf{u}$, and conserve mass. MFEMs have been successfully applied in reservoir simulation [15, 27].

2.2. Numerical methods for convection-diffusion PDEs. Standard FDMs or FEMs tend to generate solutions with severe nonphysical oscillations. In industrial applications, upstream weighting techniques are commonly used to stabilize the numerical approximations in large-scale simulators. However, these methods produce excessive numerical dispersion and spurious effects related to the grid orientation [15]. Two general classes of improved methods can be identified from the literature: the Eulerian methods that use the standard temporal discretization and the characteristic methods that carry out the temporal discretization by a characteristic tracking.

Most Eulerian methods are based on upstream weighting techniques. The optimal test function methods $[2,10]$ attempt to minimize the spatial error and yield an upstream bias in the resulting schemes. Some Eulerian methods [5] try to reduce the overall truncation error by using a nonzero spatial error to cancel temporal errors. The streamline diffusion FEMs [20, 21] add a numerical diffusion only in the direction of streamlines with no crosswind diffusion introduced. However, an a priori choice of the free parameter in the methods is not clear and is heavily problem de- 
pendent. The total variation diminishing methods (TVD), essentially nonoscillatory (ENO) methods, and other high resolution methods [11, 14, 18, 29] are well suited for nonlinear hyperbolic conservation laws and resolve shock discontinuities in the solutions without excessive smearing or spurious oscillations. These methods were extended to solve convection-diffusion PDEs [9].

Because of the hyperbolic nature of convective transport, many characteristic methods have been developed for solving convection-diffusion PDEs [4, 13, 23, 30]. Traditional forward tracking or moving mesh methods advance the grids following the characteristics and greatly reduce temporal errors. But they often severely distort the evolving grids and greatly complicate the solution procedures. The modified method of characteristics (MMOC) [13] tracks the characteristics backward from a fixed grid at the current time step and, hence, avoids the grid distortion problems present in forward tracking methods. The MMOC symmetrizes and stabilizes the convection-diffusion PDEs, greatly reduces temporal errors and so allows for large time steps in a simulation without loss of accuracy, and eliminates the excessive numerical dispersion and grid orientation effects present in many Eulerian methods $[15,27]$. However, MMOC and many other characteristic methods fail to conserve mass and have difficulties in treating general boundary conditions.

2.3. Numerical approximations to the systems (1.1)-(1.2). The combination of close couplings and nonlinearities of the governing PDEs, the singularities of the solutions due to the effect of injection and production wells, the moving steep fronts present in the solutions of the governing PDEs, the use of large grid-spacings, and the convection-diffusion feature of the governing PDEs often generate spurious numerical artifacts and inaccurate approximations to the systems. A blind linearization with little regard to the properties of the governing PDEs or the solutions can result in extremely large, ill-conditioned, nonlinear discrete algebraic systems. These issues, if not treated carefully, may destroy the usefulness of the simulation.

Fully explicit methods are computationally local and are very efficient per time step, but they often require extremely fine spatial grids and time steps and result in enormous amounts of overall computations in numerical simulations [15, 31]. Fully coupled and fully implicit methods solve all of the coupled nonlinear PDEs simultaneously in an implicit fashion, and they are unconditionally stable. There has been development of fully implicit FDM or FEM simulators for three phase flows, for steam flooding, and for compositional models [15, 27]. However, these methods are computationally expensive per time step and introduce excessive numerical diffusion and reduced accuracy with large time steps $[15,31]$. IMPES methods, which solve (1.1) implicitly for pressure and (1.2) explicitly for saturation (or concentration), provide variations to fully explicit methods to obtain better stability without increasing complexity too much. However, for difficult nonlinearities, IMPES methods are often forced to use extremely small time steps which lead to significantly compromised computational efficiency [15].

Douglas, Ewing, and Wheeler [12] presented and analyzed an efficient sequential linearization technique for the miscible displacement of one incompressible fluid by another in a porous medium. In the procedure, an MFEM was used to solve (1.1) for the pressure and Darcy velocity and a Galerkin FEM was used to solve (1.2) for the concentration. However, the use of a Galerkin FEM may generate numerical solutions with severe oscillation. Russell generalized the work in [13] and introduced the MMOC into the Society of Petroleum Engineers (SPE) literature [25] for solving (1.2) in miscible displacement of incompressible fluids in reservoir simulation, where 
the pressure equation (1.1) was solved by a biquadratic FEM. Subsequently, Ewing, Russell, and Wheeler in [12] and Russell in [25] combined the ideas and proposed an improved MMOC-MFEM sequential solution technique in the numerical simulation of the miscible displacement of incompressible fluid flows in porous media [16], in which the MMOC was used to solve (1.2) and an MFEM was employed to solve (1.1). The use of an MFEM for the pressure equation yields accurate Darcy velocity fields that conserve mass, while the application of MMOC allows large time steps to be used in the solution of the transport equation without loss of accuracy and eliminates the numerical dispersion and grid orientation effects that are among the major difficulties presented in large-scale reservoir simulators $[15,27]$.

3. An ELLAM scheme for the transport equation (1.2). The ELLAM was introduced by Celia et al. [8] for solving (one-dimensional constant-coefficient) convection-diffusion PDEs. The ELLAM formalism provides a general characteristic solution procedure for convection-dominated PDEs and a consistent framework for conserving mass and treating general boundary conditions. The ELLAM techniques symmetrize these PDEs, generate accurate numerical solutions even if large time steps are used, and eliminate nonphysical oscillations, numerical dispersion, and grid orientation artifacts. Thus, the ELLAM framework overcomes the principal shortcomings of the previous characteristic methods while maintaining their numerical advantages. In this section, we present an ELLAM scheme for the transport equation (1.2) under the assumption that the Darcy velocity $\mathbf{u}$ is known. An ELLAM-MFEM sequential solution technique for the coupled systems (1.1)-(1.2) will be described in section 5 .

3.1. A reference equation. We define a partition of the interval $[0, T]$ by

$$
0=: t_{0}^{c}<t_{1}^{c}<\cdots<t_{n}^{c}<\cdots<t_{N-1}^{c}<t_{N}^{c}:=T \quad \text { and } \quad \Delta t_{n}^{c}:=t_{n}^{c}-t_{n-1}^{c} .
$$

In the ELLAM framework, we choose the test functions $z(\mathbf{x}, t)$ to be continuous and piecewise smooth on the space-time strip $\Omega \times\left(t_{n-1}^{c}, t_{n}^{c}\right]$. We allow them to be discontinuous in time at time $t_{n-1}^{c}$ so that the ELLAM scheme can be decoupled in time. This in turn permits us to focus on the development of the scheme on the current time interval $\left[t_{n-1}^{c}, t_{n}^{c}\right]$ only and to define the test functions by constant extension along the characteristics as we should see below. We refer readers to $[1,8,31]$ for details. Multiplying (1.2) by these test functions $z(\mathbf{x}, t)$, and integrating the resulting equation over the space-time strip $\Omega \times\left(t_{n-1}^{c}, t_{n}^{c}\right]$, we obtain the following space-time weak formulation for (1.2):

$$
\begin{gathered}
\int_{\Omega} \phi(\mathbf{x}) c\left(\mathbf{x}, t_{n}^{c}\right) z\left(\mathbf{x}, t_{n}^{c}\right) d \mathbf{x}+\int_{t_{n-1}^{c}}^{t_{n}^{c}} \int_{\Omega} \nabla z(\mathbf{y}, \theta) \cdot \mathbf{D}(\mathbf{y}, \mathbf{u}(\mathbf{y}, \theta)) \nabla c(\mathbf{y}, \theta) d \mathbf{y} d \theta \\
-\int_{t_{n-1}^{c}}^{t_{n}^{c}} \int_{\Omega} c(\mathbf{y}, \theta)\left[\phi(\mathbf{y}) \frac{\partial z(\mathbf{y}, \theta)}{\partial \theta}+\mathbf{u}(\mathbf{y}, \theta) \cdot \nabla z(\mathbf{y}, \theta)\right] d \mathbf{y} d \theta \\
=\int_{\Omega} \phi(\mathbf{x}) c\left(\mathbf{x}, t_{n-1}^{c}\right) z\left(\mathbf{x}, t_{n-1}^{c,+}\right) d \mathbf{x}+\int_{t_{n-1}^{c}}^{t_{n}^{c}} \int_{\Omega} \bar{c}(\mathbf{y}, \theta) q(\mathbf{y}, \theta) z(\mathbf{y}, \theta) d \mathbf{y} d \theta
\end{gathered}
$$

where $z\left(\mathbf{x}, t_{n-1}^{c,+}\right):=\lim _{t \rightarrow t_{n-1}^{c}, t>t_{n-1}^{c}} z(\mathbf{x}, t)$ to take into account the fact that $z(\mathbf{x}, t)$ is discontinuous in time at time $t_{n-1}^{c}$. We replace the dummy variables $\mathbf{x}$ and $t$ in the space-time integrals in (3.2) by $\mathbf{y}$ and $\theta$, and we reserve $\mathbf{x}$ for the point in $\Omega$ at time $t_{n}^{c}$ or $t_{n-1}^{c}$ for later convenience. 
Different splittings of the adjoint equation of (1.2) are studied in the ELLAM framework. It is shown [8] that the test functions should be chosen to satisfy the adjoint equation of (1.2) to reflect the hyperbolic nature of (1.2):

$$
\phi(\mathbf{y}) \frac{\partial z(\mathbf{y}, \theta)}{\partial \theta}+\mathbf{u}(\mathbf{y}, \theta) \cdot \nabla z(\mathbf{y}, \theta)=0, \quad \mathbf{y} \in \bar{\Omega}, \quad \theta \in\left[t_{n-1}^{c}, t_{n}^{c}\right] .
$$

Thus, the test functions $z(\mathbf{y}, \theta)$ should be constant along the characteristics $\mathbf{y}=$ $\mathbf{r}\left(\theta ; \mathbf{x}, t_{n}^{c}\right)$, defined by the initial-value problem of the differential equation

$$
\frac{d \mathbf{r}}{d \theta}=\frac{\mathbf{u}(\mathbf{r}, \theta)}{\phi(\mathbf{r})} \quad \text { and }\left.\quad \mathbf{r}(\theta ; \overline{\mathbf{x}}, \bar{t})\right|_{\theta=\bar{t}}=\overline{\mathbf{x}}
$$

For any $(\mathbf{y}, \theta) \in \Omega \times\left[t_{n-1}^{c}, t_{n}^{c}\right]$, there exists an $\mathbf{x} \in \Omega$ such that $\mathbf{y}=\mathbf{r}\left(\theta ; \mathbf{x}, t_{n}^{c}\right)$. We apply the Euler formula at time $t_{n}^{c}$ to evaluate the source and sink term in (3.2) to obtain

$$
\begin{aligned}
\int_{t_{n-1}^{c}}^{t_{n}^{c}} & \int_{\Omega} \bar{c}(\mathbf{y}, \theta) q(\mathbf{y}, \theta) z(\mathbf{y}, \theta) d \mathbf{y} d \theta \\
& =\int_{\Omega} \int_{t_{n-1}^{c}}^{t_{n}^{c}} \bar{c}\left(\mathbf{r}\left(\theta ; \mathbf{x}, t_{n}\right), \theta\right) q\left(\mathbf{r}\left(\theta ; \mathbf{x}, t_{n}\right), \theta\right) z\left(\mathbf{x}, t_{n}\right)\left|\frac{\partial \mathbf{r}\left(\theta ; \mathbf{x}, t_{n}^{c}\right)}{\partial \mathbf{x}}\right| d \theta d \mathbf{x} \\
& =\Delta t_{n}^{c} \int_{\Omega} \bar{c}\left(\mathbf{x}, t_{n}^{c}\right) q\left(\mathbf{x}, t_{n}^{c}\right) z\left(\mathbf{x}, t_{n}^{c}\right) d \mathbf{x}+E_{q}(\bar{c}, z)
\end{aligned}
$$

where $\left|\frac{\partial \mathbf{r}\left(\theta ; \mathbf{x}, t_{n}^{c}\right)}{\partial \mathbf{x}}\right|=1+\mathcal{O}\left(t_{n}^{c}-\theta\right)$ is the Jacobian of the transformation from $\mathbf{x}$ to $\mathbf{y}=\mathbf{r}\left(\theta ; \mathbf{x}, t_{n}^{c}\right)$, and $E_{q}(\bar{c}, z)$ is the local truncation error term.

Likewise, we evaluate the diffusion-dispersion term in (1.2) to obtain

$$
\begin{aligned}
& \int_{t_{n-1}^{c}}^{t_{n}^{c}} \int_{\Omega} \nabla z(\mathbf{y}, \theta) \cdot \mathbf{D}(\mathbf{y}, \mathbf{u}(\mathbf{y}, \theta)) \nabla c(\mathbf{y}, \theta) d \mathbf{y} d \theta \\
& \quad=\Delta t_{n}^{c} \int_{\Omega} \nabla z\left(\mathbf{x}, t_{n}^{c}\right) \cdot \mathbf{D}\left(\mathbf{x}, \mathbf{u}\left(\mathbf{x}, t_{n}^{c}\right)\right) \nabla c\left(\mathbf{x}, t_{n}^{c}\right) d \mathbf{x}+E_{\mathbf{D}}(c, z),
\end{aligned}
$$

where $E_{\mathbf{D}}(c, z)$ is the local truncation error term.

Substituting (3.5) and (3.6) into (3.2), we obtain a reference equation

$$
\begin{aligned}
& \int_{\Omega} \phi(\mathbf{x}) c\left(\mathbf{x}, t_{n}^{c}\right) z\left(\mathbf{x}, t_{n}^{c}\right) d \mathbf{x}+\Delta t_{n}^{c} \int_{\Omega} \nabla z\left(\mathbf{x}, t_{n}^{c}\right) \cdot \mathbf{D}\left(\mathbf{x}, \mathbf{u}\left(\mathbf{x}, t_{n}^{c}\right)\right) \nabla c\left(\mathbf{x}, t_{n}^{c}\right) d \mathbf{x} \\
& =\int_{\Omega} \phi(\mathbf{x}) c\left(\mathbf{x}, t_{n-1}^{c}\right) z\left(\mathbf{x}, t_{n-1}^{c,+}\right) d \mathbf{x}+\Delta t_{n}^{c} \int_{\Omega} \bar{c}\left(\mathbf{x}, t_{n}^{c}\right) q\left(\mathbf{x}, t_{n}^{c}\right) z\left(\mathbf{x}, t_{n}^{c}\right) d \mathbf{x}+E(c, z)
\end{aligned}
$$

where

$$
\begin{aligned}
E(c, z):= & \int_{t_{n-1}^{c}}^{t_{n}^{c}} \int_{\Omega} c(\mathbf{y}, \theta)\left[\phi(\mathbf{y}) \frac{\partial z(\mathbf{y}, \theta)}{\partial \theta}+\mathbf{u}(\mathbf{y}, \theta) \cdot \nabla z(\mathbf{y}, \theta)\right] d \mathbf{y} d \theta \\
& -E_{\mathbf{D}}(c, z)+E_{q}(\bar{c}, z) .
\end{aligned}
$$

3.2. An ELLAM scheme. We present an ELLAM scheme for (1.2). In reservoir simulations, the physical domain is typically a rectangular domain or a finite union of rectangular domains. For simplicity, we assume $\Omega:=\left(a_{x}, b_{x}\right) \times\left(a_{y}, b_{y}\right)$ and define a tensor-product spatial partition

$$
\begin{array}{ll}
a_{x}=: & x_{0}^{c}<x_{1}^{c}<\cdots<x_{i}^{c}<\cdots<x_{I-1}^{c}<x_{I}^{c}:=b_{x}, \\
a_{y}=: & y_{0}^{c}<y_{1}^{c}<\cdots<y_{j}^{c}<\cdots<y_{J-1}^{c}<y_{J}^{c} \quad:=b_{y} .
\end{array}
$$


We define the trial and test function spaces to be the space of continuous and piecewise bilinear polynomials on the partition (3.9). Namely,

$$
S^{c}(\Omega):=M_{0,1}^{c}\left[a_{x}, b_{x}\right] \otimes M_{0,1}^{c}\left[a_{y}, b_{y}\right]
$$

where

$$
\begin{aligned}
& M_{\alpha, \beta}^{c}\left[a_{x}, b_{x}\right]:=\left\{v \in C^{\alpha}\left[a_{x}, b_{x}\right]|v|_{\left[x_{i-1}^{c}, x_{i}^{c}\right]} \in P_{\beta}\left[x_{i-1}^{c}, x_{i}^{c}\right], i=1, \ldots, I\right\}, \\
& M_{\alpha, \beta}^{c}\left[a_{y}, b_{y}\right]:=\left\{v \in C^{\alpha}\left[a_{y}, b_{y}\right]|v|_{\left[y_{j-1}^{c}, y_{j}^{c}\right]} \in P_{\beta}\left[y_{j-1}^{c}, y_{j}^{c}\right], j=1, \ldots, J\right\} .
\end{aligned}
$$

Here, $C^{0}[a, b]$ denotes the space of continuous functions on $[a, b]$ and $P_{\beta}[a, b]$ is the space of univariate polynomials of degree less than or equal to $\beta$, restricted to the interval $[a, b]$.

If we assume that the Darcy velocity $\mathbf{u}\left(\mathbf{x}, t_{n}^{c}\right)$ in (1.2) is known, then the ELLAM scheme is defined as follows: Find $c\left(\mathbf{x}, t_{n}^{c}\right) \in S^{c}(\Omega)$ such that

$$
\begin{gathered}
\int_{\Omega} \phi(\mathbf{x}) c\left(\mathbf{x}, t_{n}^{c}\right) z\left(\mathbf{x}, t_{n}^{c}\right) d \mathbf{x}+\Delta t_{n}^{c} \int_{\Omega} \nabla z\left(\mathbf{x}, t_{n}^{c}\right) \cdot \mathbf{D}\left(\mathbf{x}, \mathbf{u}\left(\mathbf{x}, t_{n}^{c}\right)\right) \nabla c\left(\mathbf{x}, t_{n}^{c}\right) d \mathbf{x} \\
=\int_{\Omega} \phi(\mathbf{x}) c\left(\mathbf{x}, t_{n-1}^{c}\right) z\left(\mathbf{x}, t_{n-1}^{c,+}\right) d \mathbf{x}+\Delta t_{n}^{c} \int_{\Omega} \bar{c}\left(\mathbf{x}, t_{n}^{c}\right) q\left(\mathbf{x}, t_{n}^{c}\right) z\left(\mathbf{x}, t_{n}^{c}\right) d \mathbf{x} \\
\forall z\left(\mathbf{x}, t_{n}^{c}\right) \in S^{c}(\Omega) .
\end{gathered}
$$

Remark 3.1. By using a characteristic tracking, the ELLAM scheme (3.12) symmetrizes the transport PDE (1.2), significantly reduces temporal errors and so generates accurate solutions even if large time steps are used in numerical simulations, and yields a 9-banded, symmetric, and positive-definite coefficient matrix. Furthermore, the ELLAM scheme conserves mass [8, 26], i.e.,

$$
\int_{\Omega} \phi(\mathbf{x}) c\left(\mathbf{x}, t_{n}^{c}\right) d \mathbf{x}=\int_{\Omega} \phi(\mathbf{x}) c\left(\mathbf{x}, t_{n-1}^{c}\right) d \mathbf{x}+\int_{t_{n-1}^{c}}^{t_{n}^{c}} \int_{\Omega} \bar{c}(\mathbf{x}, t) q(\mathbf{x}, t) d \mathbf{x} d t
$$

which is of essential importance in applications.

Remark 3.2. Because $c\left(\mathbf{x}, t_{n}^{c}\right), z\left(\mathbf{x}, t_{n}^{c}\right) \in S^{c}(\Omega)$ are standard piecewise bilinear functions at time $t_{n}^{c}$, in (3.12) all the terms except the first one on the right-hand side are standard integrals in FEMs and can be evaluated in a fairly standard way. In the first term on the right-hand side, the value of $c\left(\mathbf{x}, t_{n-1}^{c}\right)$ is known from the solution at the previous time step $t_{n-1}^{c}$. However, the test functions $z\left(\mathbf{x}, t_{n-1}^{c,+}\right):=$ $\lim _{t \rightarrow t_{n-1}^{c}, t>t_{n-1}^{c}} z(\mathbf{x}, t)=z\left(\tilde{\mathbf{x}}, t_{n}^{c}\right)$, where $\tilde{\mathbf{x}}=\mathbf{r}\left(t_{n}^{c} ; \mathbf{x}, t_{n-1}^{c}\right)$ is the point at the head of the characteristic that corresponds to $\mathbf{x}$ at the foot. The evaluation of this term becomes much more challenging in multiple dimensions, due to the multidimensional deformation of each cell $\left[x_{i-1}^{c}, x_{i}^{c}\right] \times\left[y_{j-1}^{c}, y_{j}^{c}\right]$ on which the test functions are defined as the geometry is backtracked from time step $t_{n}^{c}$ to time step $t_{n-1}^{c}$.

The most practical approach for evaluating this term is to use a forward tracking algorithm proposed by Russell and Trujillo [26]. In this algorithm, an integration quadrature is enforced at time step $t_{n-1}^{c}$ with respect to the fixed spatial grids (3.9) on which $c\left(\mathbf{x}, t_{n-1}^{c}\right)$ is defined, and the difficult evaluation is the test function $z\left(\mathbf{x}, t_{n-1}^{c,+}\right)$. Rather than backtracking the geometry and estimating the test functions by mapping the deformed geometry onto the fixed grids (3.9), discrete quadrature points chosen on the fixed grids at time step $t_{n-1}^{c}$ can be tracked forward to time step $t_{n}^{c}$, where evaluation of $z\left(\mathbf{x}, t_{n}^{c}\right)$ is straightforward. Because the forward tracking algorithm 
is used only to evaluate the first term on the right-hand side of (3.12), it has no effect on the solution grids (3.9) or the data structure of the discrete system for (3.12). Therefore, the forward tracking algorithm used here does not suffer from the complication of distorted grids, which complicates many forward tracking algorithms.

Remark 3.3. For a general velocity field $\mathbf{u}(\mathbf{x}, t)$, one cannot solve the initial-value problem (3.4) analytically to track characteristics. Hence, numerical quadratures (e.g., Euler or Runge-Kutta methods) were previously used to track characteristics in the ELLAM schemes for linear transport PDEs where $\mathbf{u}(\mathbf{x}, t)$ is assumed to be a known smooth function $[1,17,31]$. However, in the current circumstances, the velocity field $\mathbf{u}(\mathbf{x}, t)$ is given as a Raviart-Thomas MFEM solution to (1.1) (refer to section 4), which is only piecewise (cell-by-cell) smooth. Therefore, numerical quadratures would generate an inaccurate characteristic tracking, which in turn affects the accuracy of the solutions of the ELLAM schemes. In applications, within each cell the porosity $\phi(\mathbf{x})$ is constant and $u_{x}\left(\mathbf{x}, t_{n}^{c}\right)$ (or $u_{y}\left(\mathbf{x}, t_{n}^{c}\right)$ ) is linear (or constant) in the $x$ direction and constant (or linear) in the $y$ direction. Therefore, we can solve the problem (3.4) analytically to track the characteristics on a cell-by-cell basis $[17,19,28]$. In this way, we also minimize the effect of the well singularities on the characteristic tracking.

Remark 3.4. In the MMOC, which is a typical representative of many previous characteristic methods, (1.2) is rewritten in a nonconservative form

$$
\phi \frac{\partial c}{\partial t}+\mathbf{u} \cdot \nabla c-\nabla \cdot(\mathbf{D}(\mathbf{x}, \mathbf{u}) \nabla c)=(\bar{c}-c) q, \quad \mathbf{x} \in \Omega, \quad t \in[0, T],
$$

where the first equation in (1.1) has been used to get $(\nabla \cdot \mathbf{u}) c=c q$.

Then the first two terms on the left-hand side of (3.14) are combined to form one term through a characteristic tracking [13]

$$
\begin{aligned}
& \phi(\mathbf{x}) \frac{\partial c\left(\mathbf{x}, t_{n}^{c}\right)}{\partial t}+\mathbf{u}\left(\mathbf{x}, t_{n}^{c}\right) \cdot \nabla c\left(\mathbf{x}, t_{n}^{c}\right) \\
& =\left.\sqrt{\phi^{2}(\mathbf{x})+\left|\mathbf{u}\left(\mathbf{x}, t_{n}^{c}\right)\right|^{2}} \frac{d c\left(\mathbf{r}\left(t ; \mathbf{x}, t_{n}^{c}\right), t\right)}{d t}\right|_{t=t_{n}^{c}} \\
& \approx \phi(\mathbf{x}) \frac{c\left(\mathbf{x}, t_{n}^{c}\right)-c\left(\mathbf{x}^{*}, t_{n-1}^{c}\right)}{\Delta t_{n}^{c}}
\end{aligned}
$$

where

$$
\mathbf{x}^{*}:=\mathbf{x}-\frac{\mathbf{u}\left(\mathbf{x}, t_{n}^{c}\right)}{\phi(\mathbf{x})} \Delta t_{n}^{c}
$$

Substituting (3.15) for the first two terms on the left-hand side of (3.14) and integrating the resulting equation against any test functions $v(\mathbf{x}) \in S^{c}(\Omega)$, one obtains the following MMOC scheme $[13,16]$ for $(3.14)$ :

$$
\begin{aligned}
\int_{\Omega} \phi(\mathbf{x}) \frac{c\left(\mathbf{x}, t_{n}^{c}\right)-c\left(\mathbf{x}^{*}, t_{n-1}^{c}\right)}{\Delta t_{n}^{c}} v(\mathbf{x}) d \mathbf{x} & \\
& +\int_{\Omega} \nabla v(\mathbf{x}) \cdot \mathbf{D}\left(\mathbf{x}, \mathbf{u}\left(\mathbf{x}, t_{n}^{c}\right)\right) \nabla c\left(\mathbf{x}, t_{n}^{c}\right) d \mathbf{x} \\
= & \int_{\Omega}\left(\bar{c}\left(\mathbf{x}, t_{n}^{c}\right)-c\left(\mathbf{x}, t_{n}^{c}\right)\right) q\left(\mathbf{x}, t_{n}^{c}\right) v(\mathbf{x}) d \mathbf{x} \quad \forall v\left(\mathbf{x}, t_{n}^{c}\right) \in S^{c}(\Omega) .
\end{aligned}
$$

Although by (1.5) $\left.\mathbf{u}(\mathbf{x}, t) \cdot \mathbf{n}(\mathbf{x})\right|_{\Gamma}=0$, there might be some $\mathbf{x} \in \Omega$, which is close to $\Gamma$, such that the corresponding $\mathbf{x}^{*}$ determined by (3.16) runs out of the domain 
$\Omega$. This leads to implementational and analytical difficulties in the application of the MMOC. In contrast, in the ELLAM scheme (3.12) the tracking algorithm is carried out by solving the problem (3.4) analytically on a cell-by-cell basis. Since the numerical Darcy velocity $\mathbf{u}$ satisfies the no-flow boundary condition, the characteristic tracking never runs out of the domain $\Omega$ and so avoids this difficulty. This is an additional advantage of the ELLAM scheme.

4. An MFEM for the pressure equation (1.1). Because they yield an accurate velocity field $\mathbf{u}$ for porous medium flows and conserve mass, MFEMs have been widely applied in porous medium flow problems [27]. In this section, we briefly describe an MFEM for the pressure system (1.1).

4.1. Preliminary notations. Let $L^{2}(\Omega)$ be the standard function space of all the Lebesgue square integrable functions on $\Omega$. Then we define the Sobolev spaces

$$
\begin{aligned}
H^{1}(\Omega) & :=\left\{v(\mathbf{x}) \mid \frac{\partial v(x, y)}{\partial x}, \frac{\partial v(x, y)}{\partial y} \in L^{2}(\Omega)\right\}, \\
L_{0}^{2}(\Omega) & :=\left\{v(\mathbf{x}) \in L^{2}(\Omega) \mid \int_{\Omega} v(\mathbf{x}) d \mathbf{x}=0\right\}, \\
H(\operatorname{div} ; \Omega) & :=\left\{\mathbf{v}(\mathbf{x}) \in\left(L^{2}(\Omega)\right)^{2}, \nabla \cdot \mathbf{v} \in L^{2}(\Omega)\right\} \\
H_{0}(\operatorname{div} ; \Omega) & :=\{\mathbf{v}(\mathbf{x}) \in H(\operatorname{div} ; \Omega) \mid \mathbf{v}(\mathbf{x}) \cdot \mathbf{n}(\mathbf{x})=0, \mathbf{x} \in \Gamma\} .
\end{aligned}
$$

Multiplying the second equation in (1.1) by $\mu(c) \mathbf{K}^{-1}(\mathbf{x})$ yields

$$
\mu(c(\mathbf{x}, t)) \mathbf{K}^{-1}(\mathbf{x}) \mathbf{u}(\mathbf{x}, t)+\nabla p(\mathbf{x}, t)=\rho g \nabla d(\mathbf{x}), \quad \mathbf{x} \in \Omega, \quad t \in[0, T] .
$$

Integrating (4.2) against any test functions $\mathbf{v} \in H_{0}(\operatorname{div} ; \Omega)$ and applying the divergence theorem to the $\nabla p$ term, we obtain the first equation in (4.2). Then integrating the first equation in (1.1) against any test functions $w(\mathbf{x}) \in L_{0}^{2}(\Omega)$, we obtain the second equation in the following system:

$$
\begin{aligned}
\int_{\Omega} \mu(c) \mathbf{K}^{-1} \mathbf{u} \cdot \mathbf{v} d \mathbf{x}-\int_{\Omega} p \nabla \cdot \mathbf{v} d \mathbf{x} & =\int_{\Omega} \rho g \nabla d \cdot \mathbf{v} d \mathbf{x} \\
\int_{\Omega} w \nabla \cdot \mathbf{u} d \mathbf{x} & =\int_{\Omega} q(\mathbf{x}, t) w d \mathbf{x} \\
\forall \mathbf{v}(\mathbf{x}) \in H_{0}(\operatorname{div} ; \Omega) & \forall w(\mathbf{x}) \in L_{0}^{2}(\Omega), \quad t \in[0, T] .
\end{aligned}
$$

Equation (4.3) is a saddle-point problem which has been proven [6] to have a unique solution $(\mathbf{u}(\mathbf{x}, t), p(\mathbf{x}, t)) \in H_{0}(\operatorname{div} ; \Omega) \times L_{0}^{2}(\Omega) \forall t \in[0, T]$.

4.2. An MFEM for (1.1). We define the following space-time partition for the pressure system (1.1):

$$
\begin{array}{rll}
0=: & t_{0}^{p}<t_{1}^{p}<\cdots<t_{m}^{p}<\cdots<t_{M-1}^{p}<t_{M}^{p} & :=T, \\
a_{x}=: & x_{0}^{p}<x_{1}^{p}<\cdots<x_{k}^{p}<\cdots<x_{K-1}^{p}<x_{K}^{p} & :=b_{x}, \\
a_{y}=: & y_{0}^{p}<y_{1}^{p}<\cdots<y_{l}^{p}<\cdots<y_{L-1}^{p}<y_{L}^{p} & :=b_{y} .
\end{array}
$$


At each time step $t_{m}^{p}$, we define the trial and test function spaces to be the lowest order Raviart-Thomas MFEM space on the partition (4.4)

$$
\begin{aligned}
S^{p}(\Omega) & :=\left(M_{0,1}^{p}\left[a_{x}, b_{x}\right] \times M_{-1,0}^{p}\left[a_{y}, b_{y}\right]\right) \times\left(M_{-1,0}^{p}\left[a_{x}, b_{x}\right] \times M_{0,1}^{p}\left[a_{y}, b_{y}\right]\right), \\
S_{0}^{p}(\Omega) & :=\left\{\mathbf{v}(\mathbf{x}) \in S^{p}(\Omega) \mid \mathbf{v}(\mathbf{x}) \cdot \mathbf{n}(\mathbf{x})=0, \quad \mathbf{x} \in \Gamma\right\}, \\
W^{p}(\Omega) & :=M_{-1,0}^{p}\left[a_{x}, b_{x}\right] \times M_{-1,0}^{p}\left[a_{y}, b_{y}\right], \\
W_{0}^{p}(\Omega) & :=\left\{v(\mathbf{x}) \in W^{p}(\Omega) \mid \int_{\Omega} v(\mathbf{x}) d \mathbf{x}=0\right\}
\end{aligned}
$$

with

$$
\begin{aligned}
& M_{\alpha, \beta}^{p}\left[a_{x}, b_{x}\right]:=\left\{v \in C^{\alpha}\left[a_{x}, b_{x}\right]|v|_{\left[x_{k-1}^{p}, x_{k}^{p}\right]} \in P_{\beta}\left[x_{k-1}^{p}, x_{k}^{p}\right], k=1, \ldots, K\right\} \\
& M_{\alpha, \beta}^{p}\left[a_{y}, b_{y}\right]:=\left\{v \in C^{\alpha}\left[a_{y}, b_{y}\right]|v|_{\left[y_{l-1}^{p}, y_{l}^{p}\right]} \in P_{\beta}\left[y_{l-1}^{p}, y_{l}^{p}\right], l=1, \ldots, L\right\}
\end{aligned}
$$

Here, $C^{-1}[a, b]$ is the space of piecewise continuous functions. $C^{0}[a, b]$ and $P_{\beta}[a, b]$ are defined in (3.11).

If we assume that $c\left(\mathbf{x}, t_{m}^{p}\right)$ is known, an MFEM for the pressure equation (1.1) can be formulated as follows: Find $\mathbf{u}\left(\mathbf{x}, t_{m}^{p}\right) \in S^{p}(\Omega)$ and $p\left(\mathbf{x}, t_{m}^{p}\right) \in W_{0}^{p}(\Omega)$ such that

$$
\begin{aligned}
\int_{\Omega} \mu\left(c\left(\mathbf{x}, t_{m}^{p}\right)\right) \mathbf{K}^{-1} \mathbf{u}\left(\mathbf{x}, t_{m}^{p}\right) \cdot \mathbf{v} d \mathbf{x} & \\
-\int_{\Omega} p\left(\mathbf{x}, t_{m}^{p}\right) \nabla \cdot \mathbf{v} d \mathbf{x} & =\int_{\Omega} \rho g \nabla d \cdot \mathbf{v} d \mathbf{x} \quad \forall \mathbf{v}(\mathbf{x}) \in S^{p}(\Omega), \\
\int_{\Omega} w \nabla \cdot \mathbf{u}\left(\mathbf{x}, t_{m}^{p}\right) d \mathbf{x} & =\int_{\Omega} q\left(\mathbf{x}, t_{m}^{p}\right) w d \mathbf{x} \quad \forall w(\mathbf{x}) \in W_{0}^{p}(\Omega) .
\end{aligned}
$$

Remark 4.1. In the pressure system (1.1), the sharp changes of the permeability tensor $\mathbf{K}(\mathbf{x})$ and the viscosity coefficient $\mu(c)$ across fluid interfaces often lead to large changes in the pressure gradient which, in a compensatory fashion, yield a fairly smooth Darcy velocity $\mathbf{u}$. By approximating both $p$ and $\mathbf{u}$ from system (1.1) simultaneously, MFEMs generate more accurate Darcy velocities than standard FDMs or FEMs. Moreover, they conserve mass. Furthermore, the MFEM approximations to the Darcy velocity $\mathbf{u}\left(\mathbf{x}, t_{m}^{p}\right)$ are particularly suited for the semianalytical characteristic tracking used in the ELLAM scheme (3.12) and guarantee that the tracking stays within the physical domain (refer to Remarks 3.3 and 3.4).

Remark 4.2. While they possess various numerical advantages, MFEMs generate an indefinite coefficient matrix for the discrete algebraic system and are more expensive to solve than standard FDMs or FEMs. Moreover, the MFEM function spaces for $p$ and $\mathbf{u}$ must be chosen carefully, so that they satisfy the inf-sup stability condition. Extensive research has been conducted on the efficient solution of MFEM discrete systems and on the development of various generalized MFEM spaces [7]. Furthermore, an additional numerical difficulty for the MFEMs for porous medium flows is the effect of the singular source and sink terms $q(\mathbf{x}, t)$ in $(4.7)$ (see [15, 27]).

5. An ELLAM-MFEM sequential solution technique for the systems (1.1)-(1.2). Because of the couplings and nonlinearities of the PDEs in the systems (1.1)-(1.2), the effect of the point sources and sinks, the use of large grid-spacings, and the convection-diffusion feature of (1.2), fully coupled and fully implicit methods are commonly used in large-scale simulators for the systems (1.1)-(1.2). However, they are computationally expensive per time step and introduce excessive numerical diffusion 
and greatly reduced accuracy with large time steps. Since its introduction by Douglas, Ewing, and Wheeler [12], the MFEM sequential solution technique has proven to be an efficient procedure for the simulation of miscible fluid flow processes in porous media. The MMOC-MFEM solution technique proposed by Ewing, Russell, and Wheeler [16] eliminates the possible nonphysical oscillations in the MFEM sequential techniques and allows larger time steps to be used in the simulation of the systems (1.1)-(1.2). However, the MMOC-MFEM sequential method fails to conserve mass and has difficulties in treating boundary conditions (recall Remark 3.4).

In this section, we present an ELLAM-MFEM solution technique for the systems (1.1)-(1.2), in which we use the ELLAM scheme (3.12) for (1.2) and the MFEM (4.7) for the pressure system (1.1). In the miscible displacement of incompressible fluid flow processes in a porous medium reservoir, the Darcy velocity field $\mathbf{u}(\mathbf{x}, t)$ often changes less rapidly than the concentration, even if characteristics are taken into account. Therefore, it is appropriate to use coarser spatial grids and time steps (4.4) for the pressure system (1.1) than the spatial grids (3.9) and time steps (3.1) for (1.2). We assume that the spatial grids (3.9) and the time steps (3.1) are obtained by subdividing the spatial grids and the time steps (4.4). Namely, there exist

$$
\begin{aligned}
& 0=: N_{0}<N_{1}<\cdots<N_{m}<\cdots<N_{M-1}<N_{M}:=N, \\
& 0=: I_{0}<I_{1}<\cdots<I_{k}<\cdots<I_{K-1}<I_{K}:=I, \\
& 0=: J_{0}<J_{1}<\cdots<J_{l} \quad<\cdots<J_{L-1}<J_{L}:=J,
\end{aligned}
$$

such that

$$
t_{N_{m}}^{c}=t_{m}^{p}, \quad x_{I_{k}}^{c}=x_{k}^{p}, \quad y_{J_{l}}^{c}=y_{l}^{p}, \quad 0 \leq m \leq M, 0 \leq k \leq K, 0 \leq l \leq L .
$$

For $n=N_{m-1}+1, N_{m-1}+2, \ldots, N_{m}$, the concentration time step $t_{n}^{c}$ relates to the pressure time steps by $t_{m-1}^{p}<t_{n}^{c} \leq t_{m}^{p}$. Thus, we require a velocity approximation for the ELLAM scheme (3.12) based on $\mathbf{u}\left(\mathbf{x}, t_{m}^{p}\right)$ and earlier values. We define a velocity approximation by an extrapolation

$$
\begin{aligned}
(E \mathbf{u})\left(\mathbf{x}, t_{n}^{c}\right):= & \left(1+\frac{t_{n}^{c}-t_{m-1}^{p}}{t_{m-1}^{p}-t_{m-2}^{p}}\right) \mathbf{u}\left(\mathbf{x}, t_{m-1}^{p}\right)-\frac{t_{n}^{c}-t_{m-1}^{p}}{t_{m-1}^{p}-t_{m-2}^{p}} \mathbf{u}\left(\mathbf{x}, t_{m-2}^{p}\right), \\
& n=N_{m-1}+1, N_{m-1}+2, \ldots, N_{m}, \quad m=2,3, \ldots, M \\
(E \mathbf{u})\left(\mathbf{x}, t_{n}^{c}\right):= & \mathbf{u}(\mathbf{x}, 0), \quad n=1,2, \ldots, N_{1}, \quad m=1 .
\end{aligned}
$$

Equivalently, the first equation in (5.3) can be rewritten as

$$
\begin{aligned}
(E \mathbf{u})\left(\mathbf{x}, t_{n}^{c}\right):= & \frac{t_{m}^{p}-t_{n}^{c}}{t_{m}^{p}-t_{m-1}^{p}} \mathbf{u}\left(\mathbf{x}, t_{m-1}^{p}\right)+\frac{t_{n}^{c}-t_{m-1}^{p}}{t_{m}^{p}-t_{m-1}^{p}}(E \mathbf{u})\left(\mathbf{x}, t_{m}^{p}\right), \\
& n=N_{m-1}+1, N_{m-1}+2, \ldots, N_{m}, \quad m=2,3, \ldots, M,
\end{aligned}
$$

where $(E \mathbf{u})\left(\mathbf{x}, t_{m}^{p}\right)$ is evaluated by (5.3) with $t_{n}^{c}=t_{m}^{p}$ or $n=N_{m}$.

We are now in a position to present an ELLAM-MFEM sequential decoupling and linearization solution technique for the coupled systems (1.1)-(1.2). 


\section{Initialization:}

for $m=0$ and $n=0$ do

A1: Define $c(\mathbf{x}, 0)$ to be the $L^{2}$-projection of $c_{0}(\mathbf{x})$, which is given in (1.6), to the finite element subspace $S^{c}(\Omega)$ : find $c(\mathbf{x}, 0) \in S^{c}(\Omega)$, such that

$$
\int_{\Omega} c(\mathbf{x}, 0) v(\mathbf{x}) d \mathbf{x}=\int_{\Omega} c_{0}(\mathbf{x}) v(\mathbf{x}) d \mathbf{x} \quad \forall v(\mathbf{x}) \in S^{c}(\Omega) .
$$

A2: With $c(\mathbf{x}, 0)$ obtained from (5.5), find $\mathbf{u}(\mathbf{x}, 0) \in S^{p}(\Omega)$ and $p(\mathbf{x}, 0) \in W_{0}^{p}(\Omega)$, such that

$$
\begin{aligned}
\int_{\Omega} \mu(c(\mathbf{x}, 0)) \mathbf{K}^{-1} \mathbf{u}(\mathbf{x}, 0) \cdot \mathbf{v} d \mathbf{x} & \\
-\int_{\Omega} p(\mathbf{x}, 0) \nabla \cdot \mathbf{v} d \mathbf{x} & =\int_{\Omega} \rho g \nabla d \cdot \mathbf{v} d \mathbf{x} \quad \forall \mathbf{v}(\mathbf{x}) \in S^{p}(\Omega), \\
\int_{\Omega} w \nabla \cdot \mathbf{u}(\mathbf{x}, 0) d \mathbf{x} & =\int_{\Omega} q(\mathbf{x}, 0) w d \mathbf{x} \quad \forall w(\mathbf{x}) \in W_{0}^{p}(\Omega) .
\end{aligned}
$$

end do

for $m=1,2, \ldots, M$ do

B1: for $n=N_{m-1}+1, N_{m-1}+2, \ldots, N_{m}$ do

-Find $c\left(\mathbf{x}, t_{n}^{c}\right) \in S^{c}(\Omega)$, such that for $(E \mathbf{u})\left(\mathbf{x}, t_{n}^{c}\right)$ given in (5.3)

$$
\begin{gathered}
\int_{\Omega} \phi(\mathbf{x}) c\left(\mathbf{x}, t_{n}^{c}\right) z\left(\mathbf{x}, t_{n}^{c}\right) d \mathbf{x}+\Delta t_{n}^{c} \int_{\Omega} \nabla z\left(\mathbf{x}, t_{n}^{c}\right) \cdot \mathbf{D}\left(\mathbf{x},(E \mathbf{u})\left(\mathbf{x}, t_{n}^{c}\right)\right) \nabla c\left(\mathbf{x}, t_{n}^{c}\right) d \mathbf{x} \\
=\int_{\Omega} \phi(\mathbf{x}) c\left(\mathbf{x}, t_{n-1}^{c}\right) z\left(\mathbf{x}, t_{n-1}^{c,+}\right) d \mathbf{x}+\Delta t_{n}^{c} \int_{\Omega} \bar{c}\left(\mathbf{x}, t_{n}^{c}\right) q\left(\mathbf{x}, t_{n}^{c}\right) z\left(\mathbf{x}, t_{n}^{c}\right) d \mathbf{x} \\
\forall \mathbf{z}\left(\mathbf{x}, t_{n}^{c}\right) \in S^{c}(\Omega) .
\end{gathered}
$$

\section{end do}

B2: Since $t_{N_{m}}^{c}=t_{m}^{p}$ and $c\left(\mathbf{x}, t_{m}^{p}\right)=c\left(\mathbf{x}, t_{N_{m}}^{c}\right)$ is obtained from (5.7) with $n=N_{m}$, solve the following equation for $\mathbf{u}\left(\mathbf{x}, t_{m}^{p}\right) \in S^{p}(\Omega)$ and $p\left(\mathbf{x}, t_{m}^{p}\right) \in W_{0}^{p}(\Omega)$ :

$$
\begin{aligned}
\int_{\Omega} \mu\left(c\left(\mathbf{x}, t_{m}^{p}\right)\right) \mathbf{K}^{-1} \mathbf{u}\left(\mathbf{x}, t_{m}^{p}\right) \cdot \mathbf{v} d \mathbf{x} & \\
-\int_{\Omega} p\left(\mathbf{x}, t_{m}^{p}\right) \nabla \cdot \mathbf{v} d \mathbf{x} & =\int_{\Omega} \rho g \nabla d \cdot \mathbf{v} d \mathbf{x} \quad \forall \mathbf{v}(\mathbf{x}) \in S^{p}(\Omega), \\
\int_{\Omega} w \nabla \cdot \mathbf{u}\left(\mathbf{x}, t_{m}^{p}\right) d \mathbf{x} & =\int_{\Omega} q\left(\mathbf{x}, t_{m}^{p}\right) w d \mathbf{x} \quad \forall w(\mathbf{x}) \in W_{0}^{p}(\Omega) .
\end{aligned}
$$

end do

Remark 5.1. The ELLAM-MFEM solution technique inherits all the numerical advantages of the ELLAM schemes for linear transport PDEs and the MMOC-MFEM sequential method, while overcoming the shortcomings of the MMOC-MFEM method. It significantly reduces the temporal errors and thus generates accurate numerical solutions even if very large time steps $\Delta t_{n}^{c}$ are used. It symmetrizes (1.2) and yields a symmetric and positive-definite coefficient matrix with a condition number of $\mathcal{O}(1+$ $\left.|\mathbf{D}| \Delta t_{n}^{c} / h_{c}^{2}\right)$, where

$$
\begin{array}{rll}
h_{c} & :=\min _{\{i=1, \ldots, I ; j=1, \ldots, J\}} & \left\{\Delta x_{c}:=x_{i}^{c}-x_{i-1}^{c}, \Delta y_{c}:=y_{j}^{c}-y_{j-1}^{c}\right\}, \\
h_{p}:=\min _{\{k=1, \ldots, K ; l=1, \ldots, L\}} & \left\{\Delta x_{p}:=x_{k}^{p}-x_{k-1}^{p}, \Delta y_{p}:=y_{l}^{p}-y_{l-1}^{p}\right\} .
\end{array}
$$


Remark 5.2. The MFEM (4.7) has an indefinite symmetric coefficient matrix with a condition number of $\mathcal{O}\left(h_{p}^{-2}\right)$. Hence, it is much more expensive to solve than the ELLAM scheme (3.12), even if preconditioning techniques are used. On the other hand, by utilizing the flow property that the Darcy velocity field $\mathbf{u}$ changes less rapidly than the concentration $c$, the ELLAM-MFEM (as well as the MFEM-based and the MMOC-MFEM) solution technique allows much coarser spatial grids and time steps for the pressure system (1.1) to be used in a simulation without loss of accuracy. The application of the coarser spatial grids for the pressure reduces the size of the coefficient matrix and therefore its condition number, while the use of coarser time steps reduces the number of times the MFEM (4.7) needs to be solved. In this way, the ELLAM-MFEM sequential technique further enhances the computational efficiency.

Remark 5.3. The ELLAM-MFEM solution technique could be iterated at each pressure time step $t_{m}^{p}$. This would use the computed Darcy velocity $\mathbf{u}\left(\mathbf{x}, t_{m}^{p}\right)$ from (5.8) to replace the extrapolated Darcy velocity $(E \mathbf{u})\left(\mathbf{x}, t_{m}^{p}\right)$ in (5.4) in computing $(E \mathbf{u})\left(\mathbf{x}, t_{n}^{c}\right)$. Then the new velocity $(E \mathbf{u})\left(\mathbf{x}, t_{n}^{c}\right)$ would be used in the step B1. The present sequential procedure would be considered the first iteration. This was not attempted in this study. An iterated ELLAM-MFEM sequential solution procedure will probably be needed in more complicated applications.

6. Numerical experiments. In this section, we apply the ELLAM-MFEM solution technique to a variety of two-dimensional miscible displacement problems of one incompressible fluid by another in porous media to examine its performance. The test runs include problems with large mobility ratios, anisotropic dispersion in tensor form, discontinuous permeabilities and porosities, and point sources and sinks. In the numerical experiments, we tried to choose test problems with reported data and results in the literature, to justify that the ELLAM-MFEM solution technique generates correct solutions, and to have some approximate comparisons of the ELLAM-MFEM technique with other widely used methods in terms of the spatial grids and time steps used in order to generate comparable solutions.

The numerical experiments simulated miscible displacement within a horizontal reservoir of a thickness of one unit over a period of 10 years (3600 days) for one-quarter of a regular five-spot pattern with injection and production wells at the corners. The spatial domain is $\Omega=(0,1000) \times(0,1000) \mathrm{ft}^{2}$, the time period $[0, T]=[0,3600]$ days, and the viscosity of the oil is $\mu(0)=1.0 \mathrm{cp}$. The injection well is located at the upperright corner $(1000,1000)$ of the domain with an injection rate of $q=30 \mathrm{ft}^{2} /$ day and an injection concentration of $\bar{c}=1.0$. The production well is located at the lower-left corner $(0,0)$ with a production rate of $q=30 \mathrm{ft}^{2} /$ day. The initial concentration is $c_{0}(x, y)=0$. In the numerical simulation, we use a fairly coarse uniform spatial grid of $\Delta x^{c}=\Delta y^{c}=\Delta x^{p}=\Delta y^{p}=50 \mathrm{ft}$ in both $x$ and $y$ directions, although we understand that a simulation on a nonuniform partition with finer cells around wells probably generate more accurate solutions and our simulator allows such a partition. We also take an extremely large time step of $\Delta t^{c}=\Delta t^{p}=360$ days (one year). In contrast, in the numerical results reported previously in the literature, the time steps were chosen from a few days for FDM or FEM simulators to about a month for MMOC-based simulators $[16,15,25]$.

Test 1 . We assume that the porous medium is homogeneous and isotropic. The permeability coefficients (diagonal entries of $\mathbf{K}$ ) are given by $k_{x}=k_{y}=80 \mathrm{md}$, and the porosity of the medium is specified as $\phi=0.1$. Furthermore, we assume that the mobility ratio between the resident and injected fluids is $M=1$ and that the physical diffusion-dispersion term is given by $D_{m}:=\phi d_{m}=1.0 \mathrm{ft}^{2} /$ day, $D_{l}:=\phi d_{l}=0.0 \mathrm{ft}$, 


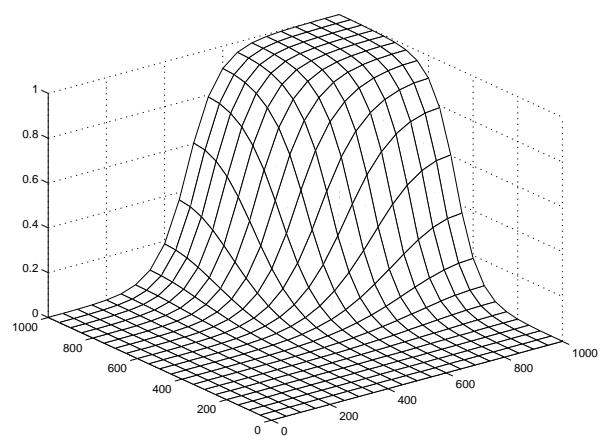

(a) Surface plot at $t=3$ years.

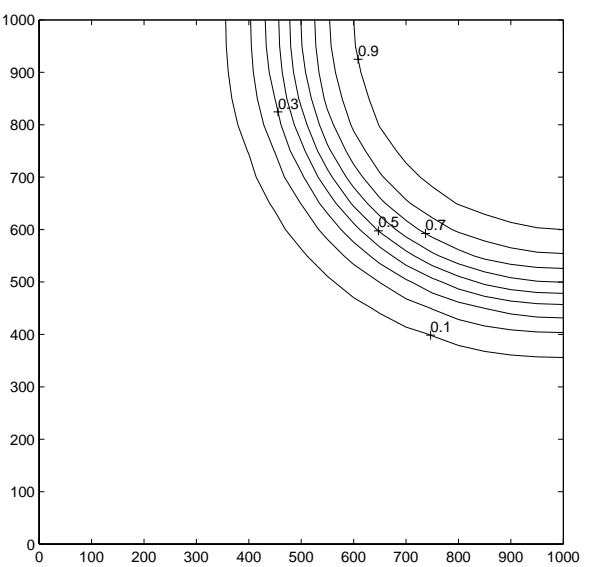

(b) Contour plot at $t=3$ years.

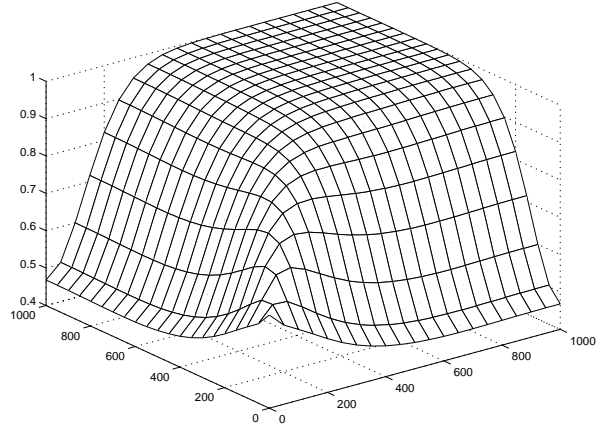

(c) Surface plot at $t=10$ years.

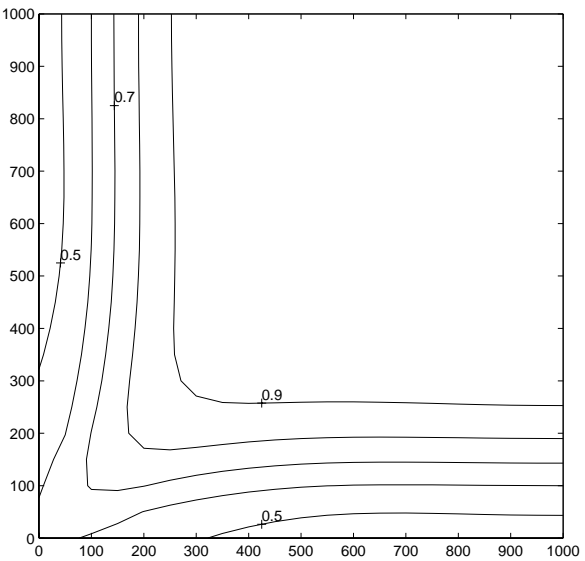

(d) Contour plot at $t=10$ years.

FIG. 1. The concentration of the invading component in Test 1 at 3 and 10 years.

and $D_{t}:=\phi d_{t}=0.0 \mathrm{ft}$. Namely, only molecular diffusion is present. This example has been widely used in testing the performance of a simulator since the qualitative behavior of its numerical simulation is understood fairly well.

The surface and contour plots for the concentration of the invading fluid at $t=$ 3 years (1080 days) are presented in Figure 1 (a) and (b). The contours of the concentration are a family of concentric circles, which are physically reasonable due to the following reasons: (i) Mobility ratio $M=1$ implies that the fluid has a constant viscosity $\mu(c)=\mu(0)$. (ii) Together with the facts that $\mathbf{K}$ is a constant tensor and that the reservoir is horizontal, we see that the Darcy velocity $\mathbf{u}$ is actually radial. (iii) Only the molecular diffusion, which is isotropic, is present. In fact, since the model does not include any permeability/viscosity variations or mechanical dispersion effects, any fingering phenomenon, if it happened, would be due to numerical errors and not to the modeling of any physics. Because of the effect of the no-flow boundary conditions and the production wells, the invading fluid moves faster along the diagonal (flow direction) of the reservoir with no fingering phenomena present. This was observed from the surface and contour plots, Figure 1 (c) and (d), of the concentration of the invading fluid at $t=10$ years (3600 days). These results demonstrate that even 
though extremely large time steps have been used in the simulation, the ELLAMMFEM simulator still generates accurate and physically reasonable solutions.

To investigate the mass conservation property of the ELLAM-MFEM solution technique, we calculate the mass balance error numerically. We divide the difference of the left-hand and right-hand sides of (3.13) by the mass $\int_{\Omega} \phi(\mathbf{x}) c\left(\mathbf{x}, t_{n}^{c}\right) d \mathbf{x}$ at the time $t_{n}^{c}$. At the production well, $\bar{c}(\mathbf{x}, t)=c(\mathbf{x}, t)$. We use a trapezoidal quadrature to evaluate the temporal integral. In the 10-year simulation, the mass balance error is $1.99 \times 10^{-4}$. With a refined time step of $\Delta t / 10$, the mass balance error is reduced to $2.05 \times 10^{-5}$. To indicate the accuracy of the ELLAM-MFEM solution technique, we compare the numerical solution with the solution obtained with a refined grid of $\Delta t / 10$ and $\Delta x / 2=\Delta y / 2$. The difference in the $L^{\infty}$ - and $L^{1}$-norms is $5.71 \times 10^{-2}$ and $4.88 \times 10^{-3}$, respectively.

Test 2. We consider a simulation with an adverse mobility ratio of $M=41$ and an anisotropic dispersion in tensor form. The physical diffusion-dispersion term is given by $D_{m}:=\phi d_{m}=0.0 \mathrm{ft}^{2} /$ day, $D_{l}:=\phi d_{l}=5.0 \mathrm{ft}$, and $D_{t}:=\phi d_{t}=0.5$ $\mathrm{ft}$. The permeability and porosity of the medium are taken to be the same as in Test 1. Equations (1.1)-(1.2) were derived via a volume-averaging mechanism and hold only on a macroscopic scale, so they do not model physical behavior on a porevolume scale. Nevertheless, by including differences in longitudinal versus transverse dispersion levels (which can be viewed as a macroscopic reflection of the microscopic mechanism), (1.1)-(1.2) should model the corresponding (microscopic) behavior of the flow in the form of a macroscopic fingering phenomenon due to varying flow velocities. The macroscopic fingering phenomenon should propagate and grow in a manner akin to viscous fingering on a smaller scale. We refer readers to [15] for detailed descriptions on these concepts.

The surface and contour plots for the concentration of the invading fluid at $t=3$ years (1080 days) and 10 years (3600 days) are presented in Figure 2 (a)-(b) and (c)-(d), respectively. Due to the effect of the large adverse mobility ratio $M=41$, the viscosity $\mu(c)$ given by the expression (1.3) changes rapidly across the steep fluid interface. Consequently, the Darcy velocity $\mathbf{u}$ has a rapid change across the fluid interface. Moreover, the large differences in longitudinal versus transverse dispersion levels force the fluid flow to move much faster along the diagonal direction (flow direction) from the injection well to the production well. The concentration front moves much faster in the diagonal direction than it did when only the molecular diffusion term was present.

Test 3. We consider the numerical simulation of a miscible displacement in a porous medium with discontinuous permeabilities, which is often encountered in many field applications. In the example run, we use the same data as in Test 2, with an exception that $k_{x}=k_{y}=80 \mathrm{md}$ is specified on the subdomain $\Omega_{L}:=(0,1000) \times$ $(0,500)$ (the lower half of the domain $\Omega$ ) and that $k_{x}=k_{y}=20$ md is given on the subdomain $\Omega_{U}:=(0,1000) \times(500,1000)$ (the upper half of the domain $\Omega$ ). The surface and contour plots for the concentration of the invading fluid at $t=3$ years (1080 days) and 10 years (3600 days) are presented in Figure 3 (a)-(b) and (c)-(d), respectively.

From Figure 3 (a)-(b), we see that the concentration front initially moves faster in the vertical direction than in the horizontal direction, because the subdomain $\Omega_{L}$ has a larger permeability and, thus, a larger Darcy velocity than the subdomain $\Omega_{U}$. Once the invading fluid reaches $\Omega_{L}$, it starts to move much faster in the horizontal direction on $\Omega_{L}$ than on $\Omega_{U}$ due to the same reason. 


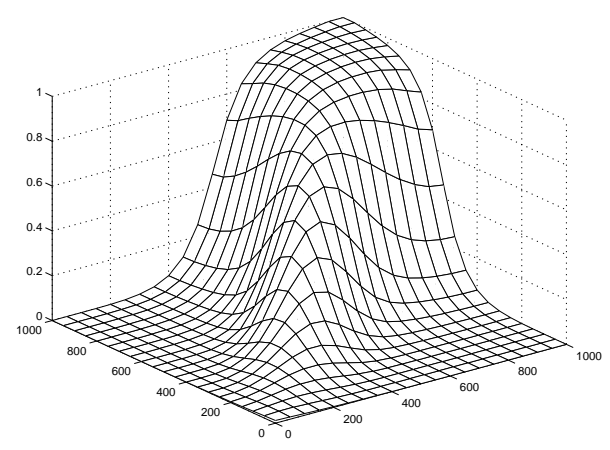

(a) Surface plot at $t=3$ years.

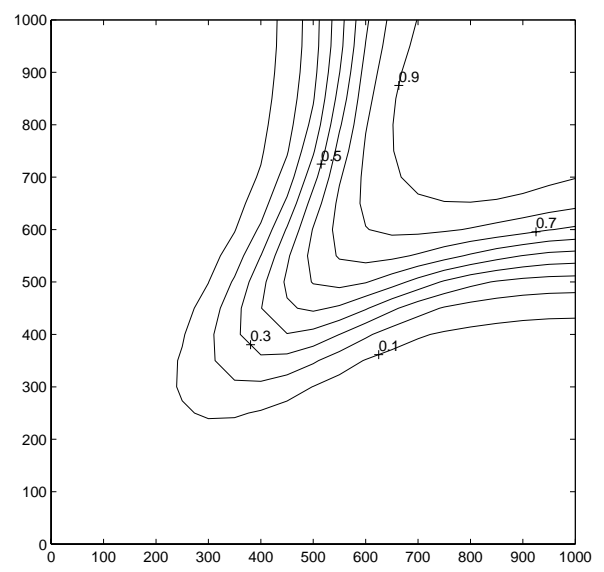

(b) Contour plot at $t=3$ years.

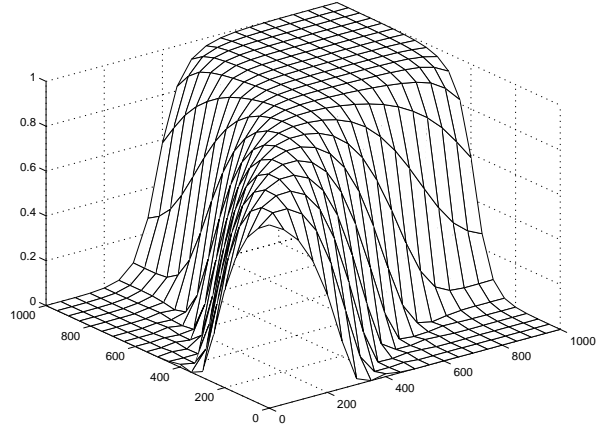

(c) Surface plot at $t=10$ years.

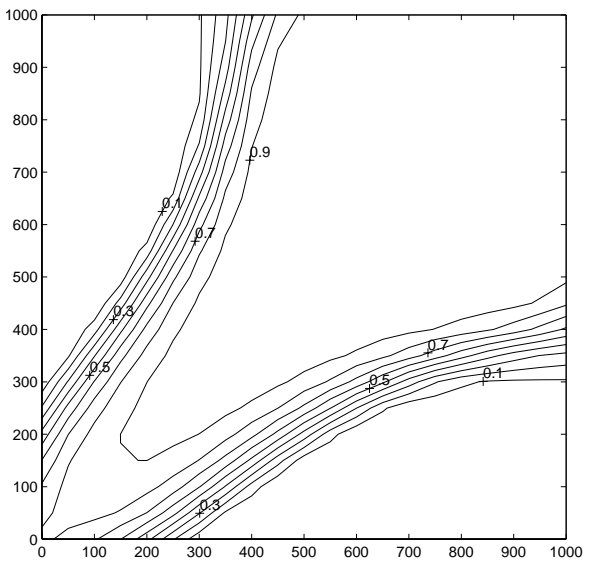

(d) Contour plot at $t=10$ years.

FIG. 2. The concentration of the invading component in Test 2 at 3 and 10 years.

Test 4 . We consider a simulation in a porous medium with piecewise structures. On the subdomain $\Omega_{I}:=(150,550) \times(150,550)$, we specify that the permeability of the medium is $k_{x}=k_{y}=25 \mathrm{md}$, that the porosity of the medium is $\phi=0.09$, and that the physical diffusion-dispersion term is $D_{m}:=\phi d_{m}=0.0 \mathrm{ft}^{2} /$ day, $D_{l}:=\phi d_{l}=4.5$ $\mathrm{ft}$, and $D_{t}:=\phi d_{t}=0.45 \mathrm{ft}$. On the subdomain $\Omega_{O}:=\Omega-\Omega_{I}$, we specify that the permeability of the medium is $k_{x}=k_{y}=80 \mathrm{md}$, that the porosity of the medium is $\phi=0.1$, and that the physical diffusion-dispersion term is $D_{m}:=\phi d_{m}=0.0 \mathrm{ft}^{2} /$ day, $D_{l}:=\phi d_{l}=5.0 \mathrm{ft}$, and $D_{t}:=\phi d_{t}=0.5 \mathrm{ft}$. The mobility ratio is still given as $M=41$. The surface and contour plots for the concentration of the invading fluid at $t=3$ years (1080 days) and 5 years (1800 days) are presented in Figure 4 (a)-(d), while the corresponding plots at $t=7$ years (2520 days) and 10 years (3600 days) are presented in Figure 4 (e)-(h).

From these numerical results, we have the following observations: (i) The ELLAMMFEM sequential solution technique can simulate fluid flows in porous media with fairly complex structures, and generates accurate and physically reasonable solutions even though a fairly coarse spatial grid and an extremely large time step are used in the simulation that in turn implies significantly improved computational efficiency. 


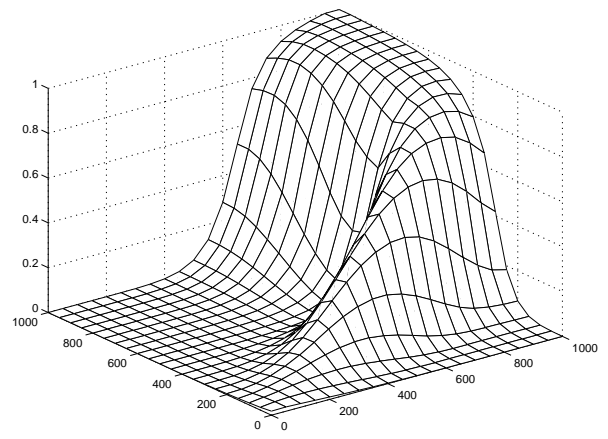

(a) Surface plot at $t=3$ years.

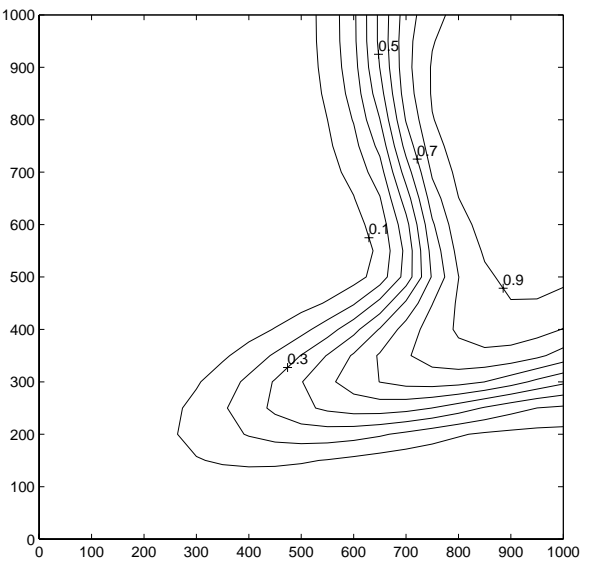

(b) Contour plot at $t=3$ years.

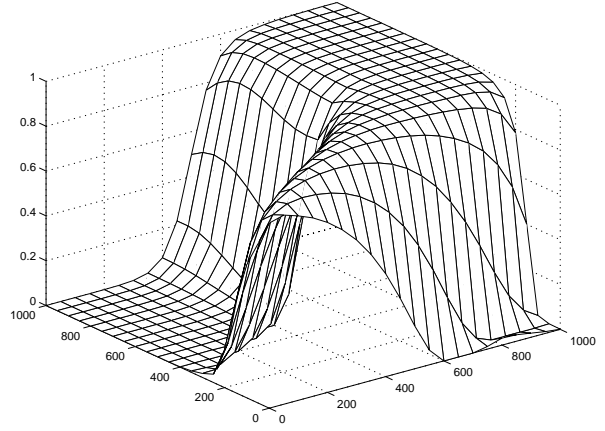

(c) Surface plot at $t=10$ years.

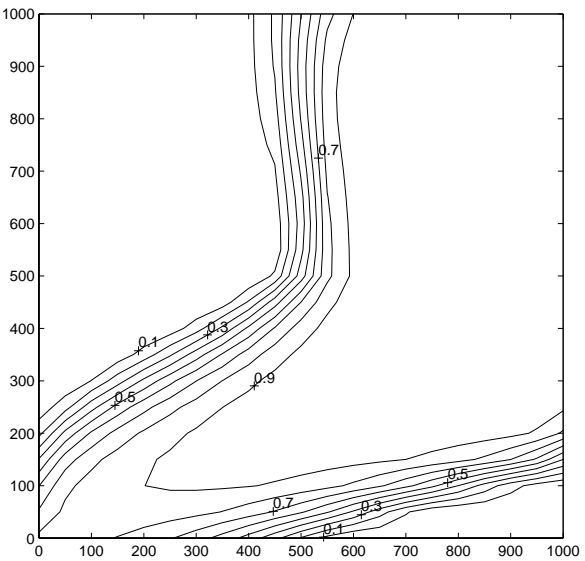

(d) Contour plot at $t=10$ years.

FIG. 3. The concentration of the invading component in Test 3 at 3 and 10 years.

(ii) Comparing these results with those in Test 2, we see that if one has a choice, one should put the production well in a low permeability zone to increase the area swept by the injected fluid. This illustrates how the results of numerical simulations could help the decision making in the petroleum reservoir industry. (iii) A very important technique in enhanced oil recovery is the use of polymers in flooding processes to alter the permeability of the reservoir porous medium to allow the fluid to flow in certain ways. Since the polymers are highly viscous, they can be used to selectively block or reduce the permeabilities of certain pores or flow regions to direct the fluid flow in a manner to optimize the hydrocarbon recovery. Test 4 could also serve as a demonstration for this technique. In this case, one can view that the original fluid and porous medium properties are given as in Test 2. By injecting some polymers in some ways, one alters the properties of the medium to those given in this test. By comparing the corresponding results in Figures 2 and 4, we see that the injected fluid in Figure 4 swept a larger area, which in turn implies a larger output. Since a major cost in the petroleum industry is the cost of drilling (injection and production) wells, these results show the effect of enhanced oil recovery. 


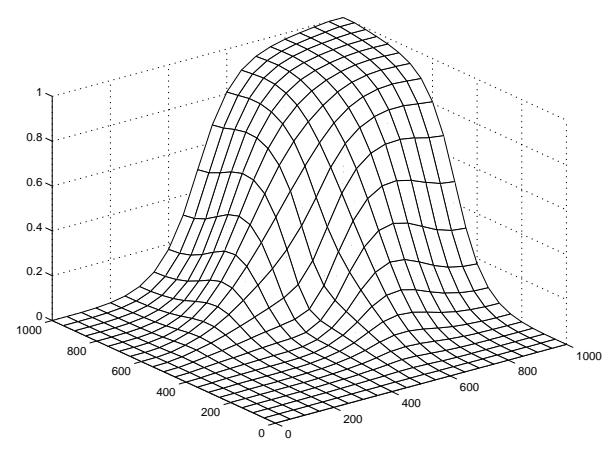

(a) Surface plot at $t=3$ years.

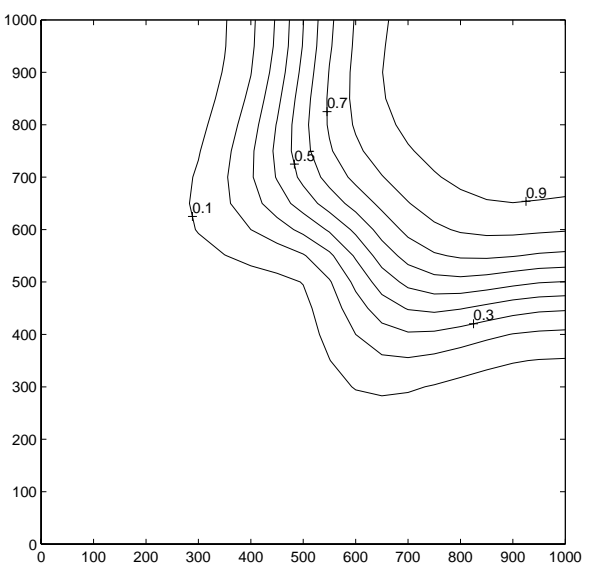

(b) Contour plot at $t=3$ years.

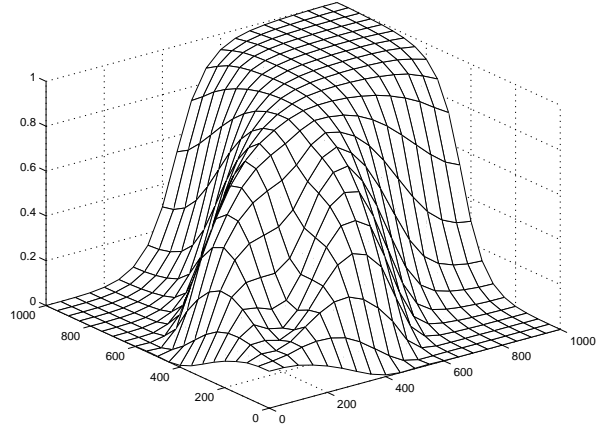

(c) Surface plot at $t=5$ years.

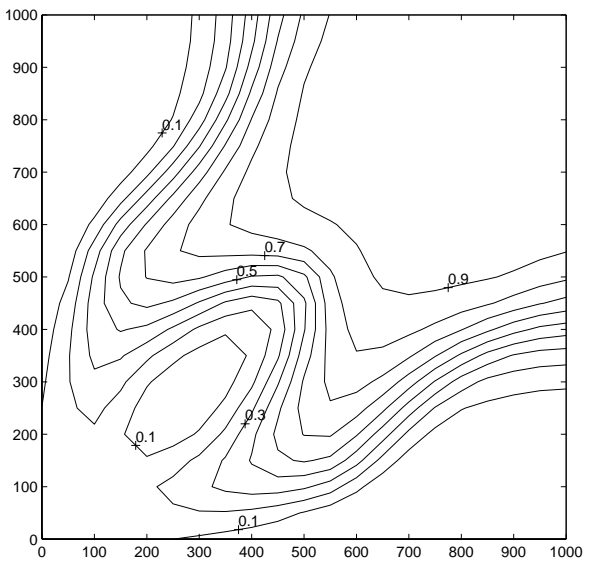

(d) Contour plot at $t=5$ years.

FIG. 4. The concentration of the invading component in Test 4 at 3 and 5 years.

7. Summary and conclusions. We develop an ELLAM-MFEM sequential solution technique for miscible fluid flows in porous media with injection and production wells (point sources and sinks), in which we use an ELLAM to solve the transport equation for concentration and an MFEM to solve the pressure equation for the pressure and Darcy velocity. The ELLAM-MFEM solution technique significantly reduces the temporal truncation errors, and thus generates accurate numerical solutions even if fairly coarse spatial grids and very large time steps are used. It symmetrizes the transport equation and greatly reduces or eliminates nonphysical oscillation and/or excessive numerical dispersion present in many large-scale simulators that are widely used in industrial applications. In this manner, the ELLAM-MFEM solution technique has a greatly improved computational efficiency over many other methods. Furthermore, the ELLAM-MFEM solution technique conserves mass and treats boundary conditions accurately, and therefore overcomes all the shortcomings of the MMOCMFEM method while maintaining its numerical advantages. 


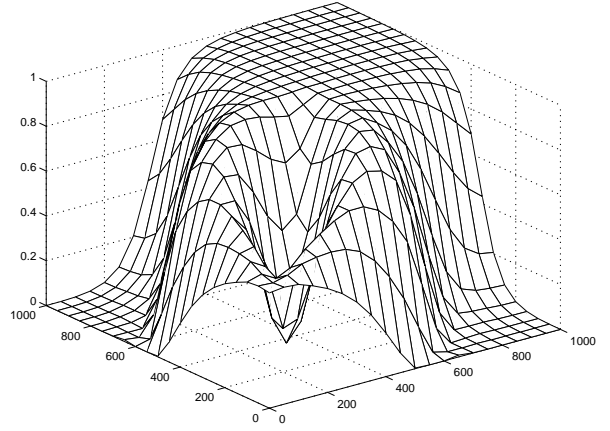

(e) Surface plot at $t=7$ years.

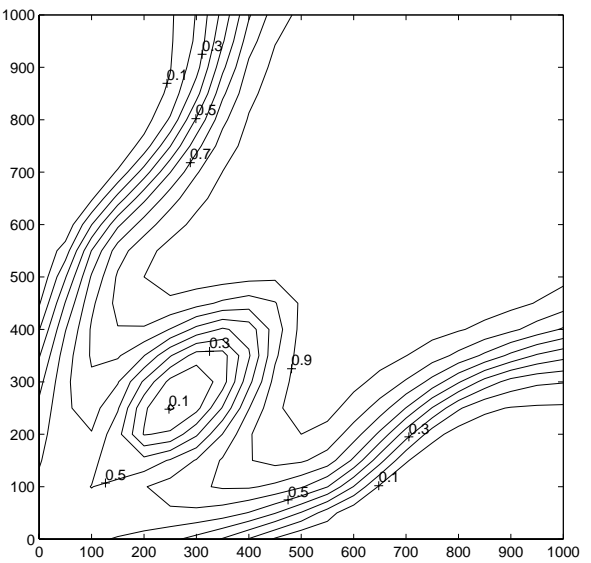

(f) Contour plot at $t=7$ years.

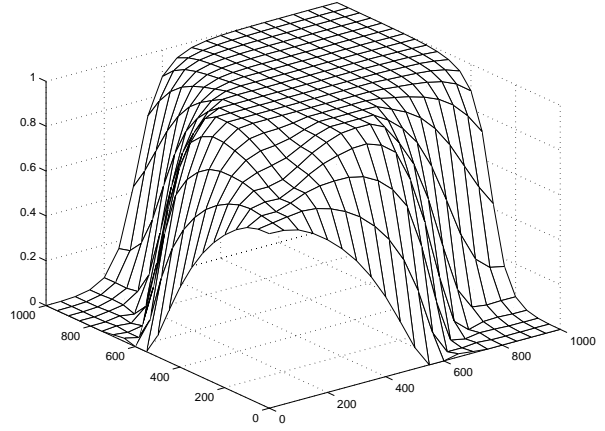

(g) Surface plot at $t=10$ years.

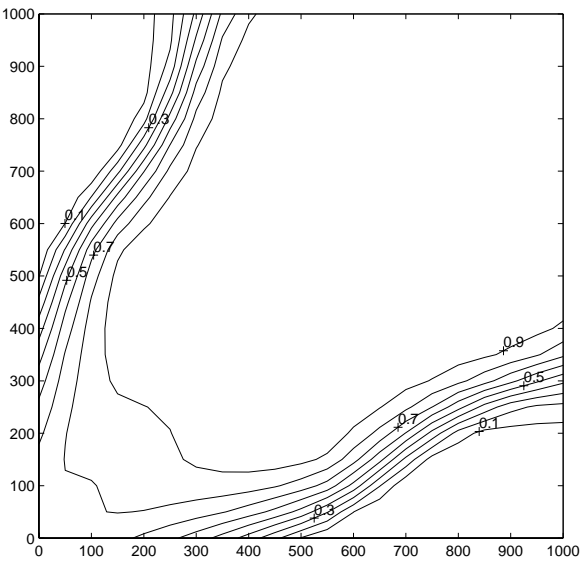

(h) Contour plot at $t=10$ years.

FIG. 4(CON'T.) The concentration of the invading component in Test 4 at 7 and 10 years.

Previous numerical experiments $[1,31]$ showed that the ELLAM often outperformed many widely used methods, such as the upwind FDM, various Galerkin and Petrov-Galerkin FEMs [2, 5, 10], the streamline diffusion FEMs [20, 21], and the MUSCL and minmod schemes $[11,14,18,29]$ in the context of linear transport PDEs. The present numerical experiments illustrate that the ELLAM-MFEM solution technique can simulate miscible displacements of incompressible fluid flows in porous media accurately with fairly coarse spatial grids as well as very large time steps, which are much larger than the time steps used in the MMOC-MFEM sequential solution procedure and one or two orders of magnitude larger than those used in many large-scale simulators. The ELLAM-MFEM technique can treat large mobility ratios, discontinuous permeabilities and porosities, anisotropic dispersion in tensor form, and point sources and sinks. 


\section{REFERENCES}

[1] M. Al-Lawatia, R.C. Sharpley, and H. Wang, Second-order characteristic methods for advection-diffusion equations and comparison to other schemes, Advances in Water Resources, 22 (1999), pp. 741-768.

[2] J.W. Barrett And K.W. Morton, Approximate symmetrization and Petrov-Galerkin methods for diffusion-convection problems, Comput. Methods Appl. Mech. Engrg., 45 (1984), pp. $97-122$.

[3] J. BEAR, Hydraulics of Groundwater, McGraw-Hill, New York, 1979.

[4] J.P. Benque And J. Ronat, Quelques difficultés des modeles numeriques en hydraulique, in Computing Methods in Applied Sciences and Engineering VII, R. Glowinski and J.-L. Lions eds., North-Holland, Amsterdam, New York, 1982, pp. 471-494.

[5] E.T. Bouloutas And M.A. Celia, An improved cubic Petrov-Galerkin method for simulation of transient advection-diffusion processes in rectangularly decomposable domains, Comput. Methods Appl. Mech. Engrg., 91 (1991), pp. 289-308.

[6] F. BREzZI, On the existence, uniqueness and approximation of saddle-point problems arising from Lagrangian multipliers, RAIRO Modél. Math. Anal. Numér., 8 (1974), pp. 129-151.

[7] F. Brezzi And M. Fortin, Mixed and Hybrid Finite Element Methods, Springer Ser. Comput. Math. 15, Springer-Verlag, New York, 1991.

[8] M.A. Celia, T.F. Russell, I. Herrera, and R.E. Ewing, An Eulerian-Lagrangian localized adjoint method for the advection-diffusion equation, Advances in Water Resources, 13 (1990), pp. 187-206.

[9] G. Chavent, G. Cohen, J. Jaffre, R. Eymard, D.R. Guerillot, and L.Weill, Discontinuous and mixed finite elements for two-phase incompressible flow, Society of Petroleum Engineers Reservoir Engineering, 5 (1990), pp. 567-575.

[10] I. Christie, D.F. Griffiths, A.R. Mitchell, And O.C. Zienkiewicz, Finite element methods for second order differential equations with significant first derivatives, Internat. J. Numer. Methods Engrg., 10 (1976), pp. 1389-1396.

[11] P. Colella, A direct Eulerian MUSCL scheme for gas dynamics, SIAM J. Sci. Statist. Comput., 6 (1985), pp. 104-117.

[12] J. Douglas, JR., R.E. Ewing, and M.F. Wheeler, A time-discretization procedure for a mixed finite element approximation of miscible displacement in porous media, RAIRO Modél. Math. Anal. Numér., 17 (1983), pp. 249-265.

[13] J. Douglas, JR. and T.F. Russell, Numerical methods for convection-dominated diffusion problems based on combining the method of characteristics with finite element or finite difference procedures, SIAM J. Numer. Anal., 19 (1982), pp. 871-885.

[14] B. Einfeldt, On Godunov-type methods for gas dynamics, SIAM J. Numer. Anal., 25 (1988), pp. 294-318.

[15] R.E. EwING ED., The Mathematics of Reservoir Simulation, Research Frontiers in Appl. Math. 1, SIAM, Philadelphia, 1983.

[16] R.E. Ewing, T.F. Russell, and M.F. Wheeler, Simulation of miscible displacement using mixed methods and a modified method of characteristics, SPE 12241, in SPE Annual Conference Proceedings, 1983, pp. 71-81.

[17] R.E. EWING AND H. WANG, Eulerian-Lagrangian localized adjoint methods for linear advection or advection-reaction equations and their convergence analysis, Comput. Mech., 12 (1993), pp. $97-121$.

[18] A. Harten, B. Engquist, S. Osher, and S. Chakravarthy, Uniformly high order accurate essentially nonoscillatory schemes, III, J. Comput. Phys., 71 (1987), pp. 231-241.

[19] R.W. HEALY AND T.F. Russell, A finite-volume Eulerian-Lagrangian localized adjoint method for solution of the advection-dispersion equation, Water Resources Research, 29 (1993), pp. 2399-2413.

[20] T.J.R. Hughes And A.N. Brooks, A multidimensional upwind scheme with no crosswind diffusion, in Finite Element Methods for Convection Dominated Flows, ADM 34, T.J.R. Hughes, ed., American Society of Mechanical Engineers (ASME), New York, 1979, pp. $19-35$.

[21] C. Johnson, A. Szepessy, And P. Hansbo, On the convergence of shock-capturing streamline diffusion finite element methods for hyperbolic conservation laws, Math. Comp., 54 (1990), pp. 107-129.

[22] S.P. Neuman, An Eulerian-Lagrangian numerical scheme for the dispersion-convection equation using conjugate space-time grids, J. Comput. Phys., 41 (1981), pp. 270-294.

[23] O. Pironneau, On the transport-diffusion algorithm and its application to the Navier-Stokes equations, Numer. Math., 38 (1982), pp. 309-332. 
[24] P.-A. Raviart and J.-M. Thomas, A mixed finite element method for second order elliptic problems, in Mathematical Aspects of Finite Element Methods, I. Galligani and E. Magenes eds., Lecture Notes in Math. 606, Springer-Verlag, Berlin, 1977, pp. 292-315.

[25] T.F. Russell, Finite elements with characteristics for two-component incompressible miscible displacement, SPE 10500, in Proceedings 6th SPE Symposium on Reservoir Simulation, New Orleans, 1982, pp. 123-135.

[26] T.F. Russell and R.V. Trujillo, Eulerian-Lagrangian localized adjoint methods with variable coefficients in multiple dimensions, in Computational Methods in Surface Hydrology, Proceedings of the 8th International Conference on Computational Methods in Water Resources, Venice, Italy, Springer-Verlag, Berlin, New York, 1990, pp. 357-363.

[27] T.F. Russell AND M.F. Wheeler, Finite element and finite difference methods for continuous flows in porous media, in The Mathematics of Reservoir Simulation, Frontiers in Appl. Math. 1, R.E. Ewing, ed., SIAM, Philadelphia, 1983, pp. 35-106.

[28] A.L. SchafER-PERINI AND J.L. Wilson, Efficient and accurate front tracking for twodimensional groundwater flow models, Water Resoures Research, 27 (1991), pp. 1471-1485.

[29] B. VAN LEER, On the relation between the upwind-differencing schemes of Godunov, EngquistOsher and Roe, SIAM J. Sci. Statist. Comput., 5 (1984), pp. 1-20.

[30] E. VAROGLU AND W.D.L. Finn, Finite elements incorporating characteristics for onedimensional diffusion-convection equation, J. Comput. Phys., 34 (1980), pp. 371-389.

[31] H. Wang, R.E. Ewing, G. Qin, S.L. Lyons, M. Al-Lawatia, and S. Man, A family of Eulerian-Lagrangian localized adjoint methods for multi-dimensional advection-reaction equations, J. Comput. Phys., 152 (1999), pp. 120-163. 